\title{
Evaluating the Therapeutic Potential of Ritlecitinib for the Treatment of Alopecia Areata
}

\author{
Hassiel Aurelio Ramírez-Marín $\mathbb{D}^{\prime}$, Antonella Tosti $\mathbb{D}^{2}$ \\ 'Department of Dermatology, Weill Cornell Medical College, New York, NY, USA; ${ }^{2}$ Dr. Philip Frost Department of Dermatology and Cutaneous \\ Surgery, University of Miami Miller School of Medicine, Miami, FL, USA \\ Correspondence: Hassiel Aurelio Ramírez-Marín, Department of Dermatology, Weill Cornell Medical College, 4I3 E 69th St., Rm 920, New York, NY, \\ I002I, USA, Tel +I 646-962-9970; +I 52618270 8982, Email hassielramirezm@gmail.com
}

\begin{abstract}
Alopecia areata (AA) is an autoimmune condition that causes patchy hair loss, affecting up to 147 million people globally. Currently, there are no treatments approved by US Food and Drug Administration (FDA) specific for AA, and there are few effective therapeutic options for widespread and persistent illness. There is an ongoing need for a treatment that demonstrates a good clinical response with a benefit-risk ratio that is suitable for long-term use, especially for patients with chronic, extensive disease. Several clinical trials and case studies that have assessed Janus kinase inhibitors have had encouraging results. Ritlecitinib, a selective JAK3/ TEC kinase inhibitor has been demonstrated to inhibit the action of signaling molecules and immune cells that are responsible for hair loss in people with alopecia areata. Furthermore, several clinical trials are investigating the utility of ritlecitinib in patients with vitiligo, rheumatoid arthritis, Crohn's disease, and ulcerative colitis. Advantages of using ritlecitinib when compared with other nonselective JAK inhibitors include avoiding JAK1/JAK2 inhibition's clinical repercussions, which include pharmacodynamic effects such as increased cholesterol and liver enzymes, and those related to JAK2 inhibition (thrombocytopenia, anemia). Treatment with Ritlecitinib $50 \mathrm{mg}$ and $30 \mathrm{mg}$ daily for 24 weeks has been shown to induce hair regrowth with a significant proportion of patients reaching SALT 20 ( $\leq 20 \%$ scalp hair loss) after six months of therapy compared to placebo. Additional research is needed for long-term effects.
\end{abstract}

Keywords: alopecia areata, ritlecitinib, PF-06651600, hair loss, skin diseases, JAK inhibitor

\section{Plain Language Summary}

Janus kinase (JAK) inhibitors inhibit the activity and response of Janus kinases and their signaling pathways which are related to several autoimmune conditions. An advantage of these drugs is that they can be found as oral formulations, unlike other treatments with similar indications such as monoclonal antibodies, which have to be taken via intramuscular shots or infused intravenously. Results from clinical trials suggest that treatment with JAK inhibitors in alopecia areata (AA) patients can induce hair to regrow. Clinical studies have shown that ritlecitinib (a selective JAK 3 inhibitor) is well tolerated and results in significant hair regrowth in patients with AA.

\section{Introduction}

Alopecia areata (AA) is an autoimmune illness that causes patchy hair loss, most usually on the scalp but occasionally on the face (eyebrows, eyelashes, beard) and other body areas. ${ }^{1,2}$ AA has an estimated global prevalence of $0.1-0.2 \% .^{3}$ AA affects up to 147 million people globally. ${ }^{4}$ AA patients encounter symptoms when immune cells target healthy hair follicles, causing hair loss. ${ }^{1,2}$ The average onset age is between 25 and 35 years, however, it can affect older adults, children, and adolescents. ${ }^{1,2}$ Around $50 \%$ of patients present with 1 or more circular patches of scalp hair loss and recover within 1 year; however, many patients relapse. ${ }^{5,6}$ Approximately half of patients have a chronic-relapsing disease that lasts more than a year, and 10-35\% eventually experience a complete loss of scalp hair (alopecia totalis) or complete loss of scalp and body hair (alopecia totalis) (alopecia universalis). ${ }^{5-9}$ For many patients, AA is associated with a poor health-related quality of life, which can lead to psychological repercussions such as anxiety and depression. ${ }^{1}$ There are 
currently no FDA or European Medicines Agency approved medications for AA, and effective therapeutic options for widespread or chronic AA are limited. ${ }^{2}$

There is an unmet need for a consistently effective treatment with a benefit-risk ratio suitable for long-term usage for severe AA, especially for patients with chronic, extensive disease. ${ }^{10}$

Ritlecitinib is the first member of a new class of covalent kinase inhibitors with remarkable selectivity for Janus kinase 3 (JAK3). Ritlecitinib has been proven in laboratory experiments to inhibit the function of signaling molecules and immune cells that cause autoimmune hair loss in patients with AA. ${ }^{11}$

\section{JAK/STAT Pathway and Alopecia Areata}

Cytokines and their downstream pathways play critical roles in immune homeostasis, as well as in the induction and regulation of immunological responses. ${ }^{12}$ To convey information from the extracellular environment to the nucleus of immune cells, type I and II cytokines rely on the Janus kinase (JAK)/signal transducer and activator of transcription (STAT) signaling pathway. ${ }^{13}$ This pathway influences processes such as immunological response, hematopoiesis, and cell cycle regulation. ${ }^{12}$ Over 50 cytokines, including IFN- and IL-15, which are linked to the immunopathology of AA function through the JAK/STAT pathway. ${ }^{12}$

The JAK family of enzymes (JAK1, JAK2, JAK3, and tyrosine kinase 2 [TYK2]) are cytoplasmic tyrosine kinases that work in pairs to promote signaling from type I/II cytokine receptors, ${ }^{14}$ after beginning signal transduction from the cell membrane, downstream within the cell, JAK enzymes then activate the signal transducer and activator of transcription (STAT), which translocates to the nucleus, where transcription occurs. Finally, JAK signals encourage immune cells to generate cytokines, resulting in the chronic inflammation that characterizes autoimmune diseases. Unregulated JAK signaling leads to certain types of bone marrow and hematologic cancers.

A study published in 2014 by Christiano et $\mathrm{al}^{15}$ showed that in mice models of AA, cytotoxic T cells were responsible for the vast majority of the initiation of the disease, which can be reversed by inhibiting the JAK/STAT pathway. ${ }^{15}$ Interleukin (IL)-15 secretion from hair follicle endothelial cells stimulates CD8+ NKG2D+ T lymphocytes, ${ }^{15}$ as a result, interferon (IFN)- $\gamma$ is secreted, which has a receptor on hair follicle epithelial cells and triggers those cells to emit additional IL-15. ${ }^{16}$ Both IL-15 and IFN- $\gamma$ signal via the JAK/STAT pathway. As a result, AA is characterized by deregulation of JAK/STAT activity, specifically the $\gamma c$ cytokine and IFN- $\gamma$ signaling pathways. ${ }^{2,15}$

Upregulation of interleukin (IL)-15 in hair follicles leads to the recruitment and activation of natural killer, gene 2Dexpressing CD81 T cells, this results in the production of interferon gamma (IFN- $\gamma$ ) with consequent loss of hair follicle immune privilege. ${ }^{15,17,18}$

In addition, Dr Christiano et al have demonstrated that patients who received oral ruxolitinib, a JAK1 and JAK2 inhibitor, have experienced near-complete hair regrowth, hinting that this tailored therapy may have clinical utility. ${ }^{15}$

\section{JAK Inhibitors}

Janus kinase (JAK) inhibitors (Table 1) are small molecules that decrease the activity and responsiveness of Janus kinases as well as their signaling pathways. JAK inhibitors have good gastrointestinal absorption; moreover, unlike biologic drugs such as monoclonal antibodies, which must be administered intramuscularly or intravenously, they can be administered orally. Numerous JAK inhibitors are currently approved for the treatment of autoimmune conditions, such as rheumatoid arthritis, psoriatic arthritis, and ulcerative colitis, disorders affecting the blood and bone marrow, such as graft-versus-host disease (GVHD), myelofibrosis, and polycythemia vera. Numerous other JAK inhibitors are also in clinical trials for similar indications.

Topical ruxolitinib $1.5 \%$ has recently been approved by FDA for the treatment of vitiligo. This drug, a JAK1/JAK2 inhibitor, is also in clinical trials as an oral treatment for psoriasis and rheumatoid arthritis. ${ }^{19}$ Tofacitinib, a JAK1/JAK2/ JAK3 inhibitor, is approved for the treatment of polyarticular course juvenile idiopathic arthritis, psoriatic arthritis, rheumatoid arthritis, and ulcerative colitis. Tofacitinib is currently in clinical trials for systemic lupus erythematosus, primary Sjogren's syndrome, Takayasu arteritis, diffuse cutaneous systemic sclerosis, and dermatomyositis. ${ }^{19}$ Baricitinib, a JAK1/ JAK2 inhibitor, is approved for rheumatoid arthritis and is currently in clinical trials for systemic lupus erythematosus, juvenile idiopathic arthritis, primary Sjogren's syndrome, and psoriasis. ${ }^{19}$ The European Union has approved upadacitinib, 
Table I JAK Inhibitors ${ }^{40,42}$

\begin{tabular}{|c|c|c|c|c|c|}
\hline Name & JAKI & JAK2 & JAK3 & Tyk2 & Others \\
\hline Ritlecitinib (PF-0665I600) & & & $++*$ & & \\
\hline Tofacitinib (CP-690550) Citrate & & ++ & ++++ & & \\
\hline Tofacitinib (CP-690550) Xeljanz ${ }^{\circledR}$ & + & ++ & ++++ & & \\
\hline AT9283 & & ++++ & ++++ & +++ & Aurora B, Aurora A, AblI (T3|5I) \\
\hline FM-38I & & & ++++ & & \\
\hline Decernotinib (VX-509) & +++ & +++ & ++++ & +++ & \\
\hline Selective JAK3 inhibitor I & + & + & ++++ & & \\
\hline Cerdulatinib (PRT062070) hydrochloride & +++ & +++ & +++ & ++++ & ARK5, MSTI, Fms \\
\hline Cerdulatinib (PRT062070) & +++ & +++ & +++ & & \\
\hline NVP-BSK805 2HCl & ++ & ++++ & +++ & +++ & \\
\hline JAK Inhibitor I (Pyridone 6) & & ++++ & +++ & ++++ & \\
\hline Gandotinib (LY2784544) & ++ & ++++ & ++ & ++ & FLT3, FLT4, FGFR2 \\
\hline ZM $39923 \mathrm{HCl}$ & + & & ++ & & TGM2, EGFR \\
\hline SAR-20347 & ++ & ++ & ++ & ++++ & IFN- $\alpha, \mid I-12$ \\
\hline Bms-9II543 & & ++++ & ++ & ++ & SET-2 \\
\hline Oclacitinib maleate & +++ & +++ & + & + & \\
\hline Momelotinib (CYT387) & +++ & +++ & + & & \\
\hline Pacritinib (SBI5|8) & & ++ & + & ++ & FLT3 (D835Y), FLT3 \\
\hline WHI-PI54 & & & + & & EGFR, Src, VEGFR \\
\hline $\mathrm{BIO}$ & & & + & ++ & GSK-3, CDK5/p35, CDK 2/Cyclin A \\
\hline XLOI9 & + & ++++ & + & & PDGFR $\beta$, FLT3 \\
\hline TGI01209 & & +++ & + & & RET, FLT3 \\
\hline Filgotinib (GLPG0634) & +++ & ++ & + & + & \\
\hline GDC046 & + & ++ & + & +++ & \\
\hline WHI-P258 & & & + & & \\
\hline JANEX-I & & & + & & \\
\hline Ruxolitinib (INCBI8424) Jakafi ${ }^{\circledR}$ & ++++ & ++++ & & & \\
\hline S-Ruxolitinib (INCB0I8424) & ++++ & ++++ & & ++ & \\
\hline Ruxolitinib phosphate & ++++ & ++++ & & & \\
\hline Baricitinib (INCB028050) Olumiant ${ }^{\circledR}$ & +++ & +++ & & ++ & \\
\hline Baricitinib phosphate & +++ & +++ & & ++ & \\
\hline Upadacitinib (ABT-494) Rinvoq ${ }^{\circledR}$ & ++ & + & & & \\
\hline Brepocitinib (PF-0670084I) & +++ & ++ & & ++ & \\
\hline
\end{tabular}


Table I (Continued).

\begin{tabular}{|l|l|l|l|l|l|}
\hline Name & JAKI & JAK2 & JAK3 & Tyk2 & Others \\
\hline AZDI48 & & ++++ & & & \\
\hline Fedratinib (TGI01348) Inrebic ${ }^{\circledR}$ & & ++++ & & & FLT3, RET \\
\hline WPI066 & & + & & & STAT3 \\
\hline AZ 960 & & ++++ & & & \\
\hline CEP-33779 & & ++++ & & & \\
\hline Ropsacitinib (PF-06826647) & + & ++ & & +++ & \\
\hline TG-89 & & + & & & \\
\hline Deucravacitinib (BMS-986I65) & & ++++ & \\
\hline Abrocitinib (PF-04965842) & ++ & + & & + & \\
\hline Solcitinib & +++ & & & & \\
\hline FLLL32 & & + & & & STAT3 \\
\hline
\end{tabular}

Notes: "+" indicates inhibitory effect. Increased inhibition is marked by a higher "+" designation. *IC50 JAK inhibition for Ritlecitinib is: $33 . \mathrm{I}$ nM. Adapted with permission from selleckchem.com. Ritlecitinib (PF-0665I600); 2021. Available from: https://www.selleckchem.com/products/pf-0665I600.html. ${ }^{40}$

Abbreviations: FLT3, Fms like tyrosine kinase 3; FLT4, Fms like tyrosine kinase 4, FGFR2, fibroblast growth factor receptor 2; RET, "rearranged during transfection", proto oncogene; EGFR, epidermal growth factor receptor; Src, "sarcoma" proto-oncogene; GSK-3, glycogen synthase kinase 3; CDK5/p35, cyclin-dependent kinase 5 activated by P35; CDK 2/Cyclin A, cyclin-dependent kinase 2/cyclin A complex; Aurora B and Aurora A, protein kinases; ARK5, AMPK-related protein kinase 5; MSTI, macrophage stimulating I; Fms, "feline mcsonough sarcoma" type II receptor tyrosine kinase; TGM2, transglutaminase 2; VEGFR, vascular endothelial growth factor; PDGFR $\beta$, plateletderived growth factor receptor beta; SET-2, Protein coding histone lysine H3-K36 methyltransferase Set2; Emt, epithelial to mesenchymal transition.

a JAK1 selective inhibitor for rheumatoid arthritis, psoriatic arthritis, and ankylosing spondylitis, it is also in clinical trials for Takayasu arteritis, giant cell arteritis, Crohn's disease, systemic lupus erythematosus, and ulcerative colitis. ${ }^{19}$ Filgotinib, a JAK1 selective inhibitor, is approved in the European Union and Japan for rheumatoid arthritis, is also being studied for Crohn's disease, ankylosing spondylitis, psoriatic arthritis, and Sjogren's syndrome. It is also being studied for Crohn's disease, ankylosing spondylitis, psoriatic arthritis, and Sjogren's syndrome. ${ }^{19}$ Peficitinib a Pan-JAK inhibitor is approved in Japan and South Korea for rheumatoid arthritis, and is also in clinical trials for psoriasis, and ulcerative colitis. ${ }^{19}$

The FDA issued a JAK inhibitor (baricitinib) Emergency Use Authorization (EUA) for use in hospitalized COVID-19 patients on November 19, $2020 .^{20}$

Janus kinase (JAK) inhibitors offer robust scientific evidence to support their usage in AA, and an increasing number of favorable clinical trials show their efficacy in hair regrowth. JAK inhibitors are already making their way into expertauthored therapy algorithms for the management of AA, despite the fact that they are not yet expressly approved for this condition. Tofacitinib (JAK1/JAK3) and ruxolitinib (JAK1/JAK2) have been demonstrated through open-label clinical trial data in individuals with AA to help reverse hair loss. ${ }^{21-23}$

In an open-label study of 66 patients, ${ }^{22}$ where the JAK1/3 inhibitor tofacitinib was administered twice daily, around one-third of patients showed $\geq 50 \%$ improvement from baseline, as determined by the severity of alopecia tool (SALT) score after three months of treatment, with side effects restricted to mild infections. ${ }^{22}$

In a retrospective analysis of 90 patients treated with tofacitinib, around $66-70 \%$ of patients achieved hair regrowth, this result depended on the dose received. ${ }^{24}$ This study also found that hair regrowth was unlikely in patients who had experienced complete or near-complete scalp hair loss for at least ten years. ${ }^{24}$ Tofacitinib may be effective in adolescents, according to another study. ${ }^{25}$

Another study with ruxolitinib, ${ }^{26}$ a JAK1/2 inhibitor, revealed that 9 out of 12 patients achieved total or nearcomplete scalp hair regrowth after 6 months of treatment. ${ }^{26}$ Furthermore, a study indicated that low-dose ruxolitinib was just as effective as higher-dose ruxolitinib for treating severe AA. ${ }^{27}$ 
The majority of JAK inhibitors under clinical development for inflammatory and autoimmune conditions are JAK1 inhibitors with different selectivity for other JAK isoforms, ${ }^{28,29}$ importantly, clinical repercussions from JAK1 inhibition include unwanted pharmacodynamic effects such as increased cholesterol and liver enzymes. ${ }^{30}$

\section{JAK3 Receptors and TEC Kinases}

Christiano et $\mathrm{a}^{31}$ used systemic and topical JAK-selective inhibitors in a mouse model of AA, and discovered that JAK1selective inhibitors as well as JAK3-selective inhibitors stimulated hair growth and reduced AA-related inflammation. ${ }^{31}$

JAK1 is broadly expressed in many tissues, and JAK3 is expressed only in hematopoietic cells. ${ }^{31}$ In preclinical models, inhibiting JAK3 signaling was shown to be sufficient for preventing and reversing AA. ${ }^{31}$ JAK3 associates with the common $\gamma c$ receptor chain shared by interleukin (IL)-2, IL-4, IL-7, IL-9, IL-15, and IL-21 receptors, and it always signals in association with JAK1. ${ }^{32}$

JAK kinases play critical roles in growth, immune response, hematopoiesis, and neural development, their inhibition has been linked to a wide range of adverse effects. The main objective of studying JAK3 inhibitors emerged of the need to find more selective drugs, with fewer side effects, that avoided those effects related to JAK2 and JAK1 inhibition.

JAK-1 inhibition has been linked to anemia, neutropenia, an increase in lipoproteins (LDL, HDL), triglycerides, liver enzymes, hemoglobin, and CPK, and most importantly an increased risk to develop infections especially upper respiratory tract infections and infectious diarrhea. ${ }^{33}$ Furthermore, erythropoietin (EPO) and thrombopoietin (TPO) rely on JAK2 for signaling, because of this, second-generation JAK inhibitors have largely avoided JAK2 inhibition. ${ }^{34,35}$

An advantage of JAK3-selective inhibition is that since it would only inhibit $\gamma \mathrm{c}$ chain receptor signaling, it would spare signaling by JAK1-dependent cytokines like IL-2, IL-6, IL-7, IL-9, IL-10, IL-11, IL-15, IL-21, IL-27, and IL-35, ${ }^{35}$ this could offer better efficacy/safety ratios. ${ }^{34,35}$

TEC kinases are activated by a variety of signals and participate in signal transduction pathways that regulate various immunological processes in both health and disease. ${ }^{34}$

Five protein kinases belong to the tyrosine kinase expressed in hepatocellular carcinoma (TEC) family, primarily expressed in hematopoietic cells: ${ }^{36,37}$ TEC, Bruton's tyrosine kinase (BTK), interleukin 2-inducible T cell kinase (ITK) (also known as TSK and EMT), resting lymphocyte kinase RLK (also known as TXK), and bone marrow tyrosine kinase on chromosome X (BMX) (also known as ETK). ${ }^{36}$ TEC kinases expressed by T cells include interleukin 2-inducible T cell Kinase (ITK), TEC, and resting lymphocyte kinase (RLK), which are activated by the T cell receptor (TCR). ${ }^{38}$ BTK is activated downstream of the $\mathrm{B}$ cell receptor (BCR) and plays a critical role in B cell development and function. ${ }^{39}$ Because they are involved in transducing signals from antigen receptors on B cells and T cells, BTK and ITK are established therapeutic targets being investigated for the treatment of inflammatory diseases. ${ }^{38,39}$

\section{Ritlecitinib Chemistry, and Basic Pharmacology}

Ritlecitinib is an orally administered selective JAK3 inhibitor, this selectivity is due to the irreversible covalent binding of ritlecitinib to Cys-909 in JAK3, which in other JAK isoforms is replaced with a serine residue ${ }^{34}$ It has a half-maximal inhibitory concentration (IC50) for JAK3 inhibition of $33.1 \mathrm{nM}$ but with no inhibitory activity (IC50>10,000 nM) the other JAK kinases (JAK1, JAK2, and TYK2), meaning that this drug has good selectivity for JAK3, sparing completely JAK1/JAK2 and TYK2 (Table 2). ${ }^{40}$ Ritlecitinib, by selectively targeting JAK3, it may spare interleukin (IL)-6 signaling as well as other JAK1-dependent immunoregulatory cytokines such as IL-10, IL-27, and IL-35. ${ }^{41}$

Ritlecitinib is the first JAK3 inhibitor with high selectivity over other JAK kinases to enter clinical trials. ${ }^{42}$

Table 2 Ritlecitinib Pharmacokinetics ${ }^{42}$

\begin{tabular}{|l|l|l|l|l|}
\hline Administration Route & Blood Clearance & Volume of Distribution & Oral Bioavailability & Systemic Half-Life \\
\hline Oral & $5.6 \mathrm{~mL} / \mathrm{min} / \mathrm{kg}$ & $1.3 \mathrm{~L} / \mathrm{kg}$ & $90 \%$ & $2 \mathrm{~h}$ \\
\hline
\end{tabular}

Note: Data from Telliez et al. ${ }^{42}$ 
Ten other kinases have a cysteine at the equivalent position of Cys-909 in JAK3, 5 of them belong to the TEC kinase family (ITK, RLK, BTK, BMX, and TEC), which are also irreversibly inhibited by ritlecitinib. ${ }^{42}$ By inhibiting TEC kinases, ritlecitinib inhibits the cytolytic function of CD8+ T cells and NK cells. ${ }^{34,42}$ Ritlecitinib inhibits Th1 and Th17 cell differentiation and function in vitro. ${ }^{31}$ Ritlecitinib inhibits the phosphorylation of STAT5 elicited by IL-2, IL-4, IL-7, and IL-15 with IC50 values of 244, 340, 407, and $266 \mathrm{nM}$, respectively, and it inhibits the phosphorylation of STAT3 elicited by IL-21 with an IC50 of $355 \mathrm{nM}^{42}$

After inhibiting BTK, ritlecitinib inhibits CD69 surface expression in B-cells, normally expressed after BCR activation with antibodies. ${ }^{42}$ Ritlecitinib also inhibits CD69 surface expression on CD4+ T cells, an effect that is most likely driven by ITK inhibition, though contributions from TEC or RLK inhibition cannot be ruled out. ${ }^{42}$

Ritlecitinib inhibits degranulation and IFN- production in CD8+ T cells, ${ }^{34}$ this drug also inhibits CD8+ T cell cytolytic functions by inhibiting ITK. Similar results have also been observed in NK cells. ${ }^{34}$

The dual activity of ritlecitinib toward JAK3 and the TEC kinase family may provide a beneficial inhibitory profile for therapeutic intervention based on the underlying pathophysiology of inflammatory diseases such as inflammatory bowel disease, rheumatoid arthritis, vitiligo, and AA. ${ }^{34,42}$

In a study performed by Robinson et $\mathrm{al}^{43}$ where 70 patients received Ritlecitinib, only 9 patients reported mild treatment-related adverse effects, among them, it was found mild lymphopenia, mild hepatotoxicity, arthralgias, peripheral edema, pruritus and 1 patient reported suicidal ideation that resolved with treatment withdrawal. ${ }^{43}$ The most common adverse effects continued to be infections and infestations, influenza occurred in $7.1 \%$ of patients, and 1 case of herpes simplex was reported. ${ }^{43}$ No patient developed bleeding events, platelet count reductions of $100 \times 10^{3} / \mathrm{mm}^{3}$, or clinically significant symptoms of anemia or hemodynamic compromise. ${ }^{43}$ Ritlecitinib performed better when evaluated for adverse effects related to cell counts, thrombopoiesis, and erythropoiesis in comparison with previously studied JAK2 inhibitors. ${ }^{43}$

The pharmacokinetics of ritlecitinib in humans are predicted to be as follows: The drug has a blood clearance of $5.6 \mathrm{~mL} /$ $\mathrm{min} / \mathrm{kg}$, a steady-state volume of distribution of $1.3 \mathrm{~L} / \mathrm{kg}$, a $90 \%$ oral bioavailability, and a systemic half-life of 2 hours. ${ }^{42}$

\section{Ritlecitinib Clinical Trials ALLEGRO Trial}

The purpose of this clinical trial was to compare the efficacy and safety profiles of ritlecitinib (PF-06651600) and brepocitinib (PF-06700841) which is an orally available selective inhibitor of TYK2 and JAK1 in subjects with alopecia areata. ${ }^{44}$ This Phase 2a, randomized, double-blind multicenter study included 14,218-75-year-old patients with moderate to severe alopecia areata ( $\geq 50 \%$ scalp hair loss), including patients with alopecia totalis and alopecia universalis. ${ }^{45}$ The primary endpoint of the study was the mean change from baseline in the SALT score at week 24, it also included an analysis over time of the SALT score change from baseline to determine the time to the first statistically significant difference from placebo, as well as a subgroup analysis to assess the impact of the duration of the current alopecia areata episode ( $<3.5$ years compared to $\geq 3.5$ years) on efficacy of ritlecitinib and brepocitinib. ${ }^{45}$ According to this phase 2 trial, ritlecitinib has an effect onset at 6 weeks. ${ }^{45}$ The 24-week response may be greater in patients who have had their current AA episode for a shorter period of time. ${ }^{45}$ Very promising data from 48 patients has already been reported by King et al this year ${ }^{11}$ with at least $50 \%$ of the patients who received ritlecitinib achieving a SALT $_{30}$ score and another $25 \%$ achieving a $\mathrm{SALT}_{90}$ score after 24 months. Eyelash improvement was reported in $81 \%$ of patients, and $72 \%$ reported eyebrow improvement. ${ }^{11}$ In this study, AASIS, an internally consistent patient-reported outcome measurement tool, was also used to assess AA-related symptoms and their impact on daily functioning. ${ }^{11}$ The improvement in AASIS scores $(-10.8[90 \% \mathrm{CI},-15.6,-5.9])$ after 24 weeks in the active treatment groups, but not in the control group, suggests a significant improvement in patients' quality of life after treatment with ritlecitinib.

Limitations of this study include the demographic representation as $69 \%$ were women and $84 \%$ of patients were white. ${ }^{11}$ This trial will have two extension periods to assess the safety and efficacy of an additional 24 weeks of ritlecitinib active treatment, crossover of therapy in nonresponders, and withdrawal of therapy and re-treatment. ${ }^{11}$ 


\section{Allegro2a Trial}

This Ritlecitinib phase 2a randomized, double-blind, placebo-controlled study to assess the safety and tolerability of ritlecitinib in adults aged 18 to 50 with AA-related scalp hair loss of $25 \%$ or more is currently ongoing globally in 39 locations including the USA, Australia, Canada, and Poland. ${ }^{46}$ It started in September 15 2020, and to this day, enrollment includes 70 participants, with an estimated completion date in May $2023{ }^{46}$ The experimental arm will receive ritlecitinib $200 \mathrm{mg}$ once daily for four weeks, followed by ritlecitinib $50 \mathrm{mg}$ daily until month 24 . At Month 9 , participants assigned to this treatment arm will also receive 3 placebo tablets for 4 weeks to keep the other arm blind. The occurrence of clinically significant abnormalities in clinical laboratory values is going to be evaluated from baseline through Month $24{ }^{46}$ This trial is also assessing the response to ritlecitinib in adults with AA using the SALT and the Patient's Global Impression of Change (PGI-C) tools, after 24 months. ${ }^{46}$

\section{Phase 2b/3 ALLEGRO Trial}

This was the first clinical trial with the aim to evaluate ritlecitinib safety and effectiveness. ${ }^{47}$

This global Phase $2 \mathrm{~b} / 3$, randomized, placebo-controlled, double-blind study looked at ritlecitinib performance in AA patients $\geq 12$ years old $(\mathrm{n}=718$ ). Patients in the study had $\geq 50 \%$ scalp hair loss and a current episode of AA that lasted 6 months to 10 years. Ritlecitinib $50 \mathrm{mg}$ or $30 \mathrm{mg}$ (with or without one month of once-daily ritlecitinib $200 \mathrm{mg}$ ), ritlecitinib $10 \mathrm{mg}$, or placebo were given to patients. ${ }^{48}$ This was followed by a 24 -week extension period in which all participants who were initially randomized to receive ritlecitinib continued on the same regimen, while participants who received placebo during the first 24 weeks advanced to one of two regimens: $200 \mathrm{mg}$ for four weeks followed by $50 \mathrm{mg}$ for 20 weeks or $50 \mathrm{mg}$ for 24 weeks. ${ }^{48}$ The $10 \mathrm{mg}$ dosing arm was evaluated for dose-ranging but not for statistically significant efficacy versus placebo. ${ }^{48}$

The primary endpoint was an absolute SALT Score $\leq 20$ at Week $24 .{ }^{49}$ When compared to placebo, a statistically significant higher proportion of patients who received ritlecitinib $30 \mathrm{mg}$ or $50 \mathrm{mg}$ once-daily, with or without a four-week initial treatment of $200 \mathrm{mg}$ once-daily, achieved an absolute SALT score of 20 after 24 weeks of treatment. ${ }^{48}$

\section{ALLEGRO LT Trial}

The Long Term ritlecitinib for the Treatment of Alopecia Areata Trial (ALLEGRO LT) is a global Phase 3 study with the goal to assess the safety and efficacy of ritlecitinib in adults and adolescents (12 years and older) with alopecia areata. The study is currently recruiting patients. It is an open-label study, all patients who enroll will receive active study drug. ${ }^{50}$

A sub-study of approximately 60 adult patients from this study will be conducted at various sites in the United States and Canada. The sub-study will assess the immune response to tetanus and meningococcal vaccines in patients who have taken $50 \mathrm{mg}$ ritlecitinib for at least 6 months. ${ }^{50}$

\section{Withdrawal/ Re-Treatment Extension Study in Patients with Alopecia Areata}

Another recent study aimed to evaluate the proportion of nonresponders from ALLEGRO trial achieving SALT 30/50/90 at week 52, the time to achieve the re-treatment criterion ( $>30 \%$ loss of the regrown hair) after withdrawal of active treatment. The mean SALT score over time among re-treated responders (Pot hoc analysis) and finally the proportion of re-treated responders achieving SALT $30 .^{51}$ It showed that patients who received placebo in the double blind (DB) treatment period and then received ritlecitinib in the extension had a response consistent with the responses of patients on active therapy in the DB treatment period. ${ }^{51}$ It also showed that the median time to meet the re-treatment criterion after withdrawing active treatment was 16 weeks for patients previously receiving ritlecitinib. Following withdrawal and retreatment, there was a loss of efficacy based on SALT 30 compared with the initial response in the DB period. SALT 30 was only achieved by $57 \%$ of patients receiving ritlecitinib and 53\% receiving PF-06700841 who had initially achieved SALT 30 on active treatment. ${ }^{51}$

Nonresponders who continued treatment for another 24 weeks were unlikely to respond during this extension study. ${ }^{51}$

Ritlecitinib continued to be generally safe and well tolerated, including in the Nonresponders group (from the initial DB period) who received 24 additional weeks of the same active treatment. ${ }^{51}$ 


\section{Contraindications and Precautions}

Due to the effect of ritlecitinib in liver enzymes, complete blood count, and the increased risk of infections, this drug should not be used in patients with an active or chronic infection; or an infection requiring hospitalization of IV antimicrobials within 6 months; special attention to avoid this drug should be given to patients with active or latent (insufficiently treated) hepatitis or tuberculosis, recurrent or disseminated Herpes Zoster, and finally, this drug should not be used when using concomitant medications associated with peripheral neurologic or hearing loss, occupational or recreational noise exposure, and $\mathrm{HbA} 1 \mathrm{c}>7.5 \%$ at screening. ${ }^{50}$

\section{Clinical Indications}

Ritlecitinib is in Phase III clinical trial for the treatment of alopecia areata and in Phase II clinical trial for the treatment of Crohn's disease, Rheumatoid arthritis, Ulcerative colitis and Vitiligo. ${ }^{48}$

In a 8-week study performed by Robinson et al in patients with rheumatoid arthritis (RA), ${ }^{43}$ treatment with ritlecitinib (200 mg once daily) was associated with significant improvements in RA disease activity and was generally well tolerated (Table 3). ${ }^{43}$

\section{Dosing}

Ritlecitinib $50 \mathrm{mg}$ and $30 \mathrm{mg}$ once daily significantly induce hair regrowth with a significant proportion of patients reaching SALT 20 (less than or equal to $20 \%$ scalp hair loss) after six months of treatment versus placebo. ${ }^{47}$

\section{Adverse Effects}

As previously stated, JAK inhibitors suppress the immune system, and their primary side effect is susceptibility to infections. Upper respiratory tract and nasal infections are the most common ones, which occur in more than $10 \%$ of patients. ${ }^{53}$ Less common infections include tuberculosis, ${ }^{52}$ herpes simplex, herpes zoster, ${ }^{53}$ and oral candidiasis.

Some patients may be at a higher risk of developing cancer. Tofacitinib, a non-selective JAK1/JAK2/JAK3 inhibitor, for example, inhibits the signaling of several cytokines, including type I and type II interferons, and reduces the number of NK cells, both of which are important in the elimination of transformed cells in cancer. ${ }^{54,55}$ From 5677 adult patients who participated in Phase II, phase III, and long-term studies of tofacitinib, 107 patients developed malignancies, the most common were lung cancer $(n=24)$, breast cancer $(n=19)$, lymphomas $(n=10)$, and gastric cancer $(n=6) .{ }^{55}$ With the development of JAK3 inhibitors, the JAK1 inhibition-related neoplasms could diminish, although the data from longterm studies are still missing. ${ }^{56,57}$

JAK inhibitors may affect cholesterol levels, $(\text { Table } 4)^{58}$ and the health-care provider may need to prescribe a statin drug. Blood clots can form, increasing the likelihood of cardiovascular events, pulmonary embolism, and deep vein thrombosis. ${ }^{59}$ Liver damage is also a possible adverse reaction with JAK inhibitor use, and finally, these drugs are contraindicated in patients with diverticulitis, as they can lead to viscous perforation. ${ }^{56,57}$

The FDA recently issued a warning about the increased risk of heart-related events, such as heart attack or stroke, as well as cancer and death, associated with the use of tofacitinib $\left(\right.$ Xeljanz $^{\circledR}$ ), baricitinib $\left(\right.$ Olumiant ${ }^{\circledR}$ ), and upadacitinib (Rinvoq $\left.{ }^{\circledR}\right){ }^{60}$

Table 3 Ritlecitinib Profile

\begin{tabular}{|c|c|c|c|c|}
\hline $\begin{array}{l}\text { Mechanism } \\
\text { of Action }\end{array}$ & Effects & Clinical Use & $\begin{array}{l}\text { Recommended } \\
\text { Dose }\end{array}$ & Toxicity, Drug Interactions \\
\hline $\begin{array}{l}\text { Selective } \\
\text { inhibition of } \\
\text { JAK3 and } \\
\text { TEC kinases. }\end{array}$ & $\begin{array}{l}\text { Inhibition of the cytolytic function of } \\
\text { CD8+ T cells and NK cells. Inhibits Th I } \\
\text { and ThI } 7 \text { cell differentiation and } \\
\text { function, inhibits } \gamma \mathrm{C} \text { cytokine signaling, } \\
\text { inhibiting the phosphorylation of STAT5 } \\
\text { elicited by IL-2, IL-4, IL-7, and IL-I5. }\end{array}$ & 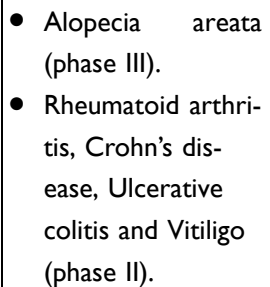 & $\begin{array}{l}\text { - Alopecia } \\
\text { Areata: 30/ } \\
50 \text { mg QD } \\
\text { - Rheumatoid } \\
\text { Arthritis: } \\
200 \text { mg QD. }\end{array}$ & $\begin{array}{l}\text { Nasopharyngitis, headache, acne, upper } \\
\text { respiratory tract infection, diarrhea, } \\
\text { nausea, neutrophil count decreased, } \\
\text { angioedema, blood creatine } \\
\text { phosphokinase increased. }\end{array}$ \\
\hline
\end{tabular}


Table 4 JAK Inhibitors Adverse Effects ${ }^{33}$

\begin{tabular}{|l|l|}
\hline $\begin{array}{l}\text { JAK } \\
\text { Isomer }\end{array}$ & Side Effects \\
\hline JAKI & $\begin{array}{l}\text { Laboratory findings: increased HDL and LDL, increase in hemoglobin, CPK elevation, hypertriglyceridemia, elevated liver enzymes. } \\
\text { Decreased reticulocytes, neutrophils, and platelet count; anemia. } \\
\text { Other adverse effects: increase in infections, neoplasias, diarrhea, nausea, acne. }\end{array}$ \\
\hline JAK2 & $\begin{array}{l}\text { Laboratory findings: Anemia, thrombocytopenia. } \\
\text { Other adverse effects: gastrointestinal effects (diarrhea, nausea). }\end{array}$ \\
\hline JAK3 & $\begin{array}{l}\text { Laboratory findings: Increased creatinine, liver transaminases, and lipid levels. Neutropenia } \\
\text { Other adverse effects: Infections }\end{array}$ \\
\hline TYK2 & $\begin{array}{l}\text { Laboratory findings: decreased neutrophils, reticulocytes, and platelet count. } \\
\text { Other adverse effects: infections (upper respiratory tract infections), headaches, gastrointestinal (diarrhea, nausea) }\end{array}$ \\
\hline
\end{tabular}

Note: Data from Jo et al. ${ }^{33}$

Abbreviations: FDA, Food and Drug Administration; AA, alopecia areata; JAK3, Janus kinase 3; TEC, tyrosine-kinase expressed in hepatocellular carcinoma, SALT, Severity of Alopecia Tool; PGI-C, Patient's Global Impression of Change; QD, once per day; IC50, half maximal inhibitory concentration; BAEP, brainstem auditory evoked potential; $\gamma c$, cytokine receptor $\gamma$ c; TYK2, tyrosine kinase 2; STAT, signal transducer and activator of transcription; DB, double blind; Nm, nanomolar; CAS, Chemical Abstracts Service; TEC, the tyrosine kinase expressed in hepatocellular carcinoma family of protein kinases; BTK, Bruton's tyrosine kinase; BMX, bone marrow tyrosine kinase on chromosome X; ITK, interleukin 2-inducible T cell kinase; RLK, resting lymphocyte kinase; FLT3, Fms like tyrosine kinase 3; FLT4, Fms like tyrosine kinase 4, FGFR2, fibroblast growth factor receptor 2; TCR, T cell receptor; MHC, major histocompatibility complex; CXCL, chemokine ligand; RET, "rearranged during transfection", proto oncogene; EGFR, epidermal growth factor receptor; Src, "sarcoma" protooncogene; GSK-3, glycogen synthase kinase 3; CDK5/p35, cyclin-dependent kinase 5 activated by p35; CDK 2/Cyclin A, cyclin-dependent kinase 2/cyclin A complex; Aurora B and Aurora A, protein kinases; ARK5, AMPK-related protein kinase 5; NKG2D, NK cell receptor D; P, phosphorylated; MSTI, macrophage stimulating I; Fms, "feline mcsonough sarcoma" type II receptor tyrosine kinase; TGM2, transglutaminase 2; VEGFR, vascular endothelial growth factor; PDGFR $\beta$, platelet-derived growth factor receptor beta; SET-2, Protein coding histone lysine H3-K36 methyltransferase Set2; ULN, upper limit of normal; IP- I0, Interferon- $\gamma$-Inducible Protein I0; AASIS, Alopecia Areata Symptom Impact Scale. IL, interleukin; INF$\gamma$, interferon-gamma; IFN $\gamma R$, interferon-gamma receptor; IL/LI5R 3 , IL2/ILI5 receptor subunit beta; ILI5R $\alpha$, ILI5 receptor subunit alpha.

Data with ritlecitinib show that it does not have some of the side effects associated with JAK1 inhibitors, in particular changes in the lipid profile. ${ }^{38}$

The study presented by King et al showed that $67 \%$ of patients that received ritlecitinib reported at least one adverse effect. $^{11}$ The most common adverse events (AEs) observed in the study were headache (5/48 [10\%]), nasopharyngitis (5/ $48[10 \%])$, and upper respiratory tract infection $(4 / 48[8 \%]) .{ }^{48}$ Other common side effects include acne $(5 / 48[10 \%])$, and nausea. There were no clinically significant differences from baseline in electrocardiogram findings, hematology tests, or vital signs, ${ }^{11} 1$ patient had a decreased lymphocyte count. ${ }^{11} 2$ patients discontinued ritlecitinib due to an adverse effect, among them: 1 patient (1/48 [25]) developed angioedema and the other one (1/48 [25]) an increased level of serum creatine phosphokinase. ${ }^{61} 8$ patients developed mild to moderate herpes zoster. ${ }^{11}$ In the ritlecitinib 50 mg group, there was one case of pulmonary embolism. ${ }^{11} 2$ malignancies (both breast cancers) reported in the ritlecitinib $50 \mathrm{mg}$ group were reported, which occurred on Days 68 and 195, the participation of these patients in the study was discontinued. ${ }^{48}$ The trial had no major adverse cardiac events (MACE), deaths, or opportunistic infections. ${ }^{11}$

\section{Conclusion}

Treatment with 30-50 mg once daily of the JAK3/TEC inhibitor ritlecitinib for 24 weeks has been shown to result in clinically significant hair regrowth in patients who have AA with $>50 \%$ scalp hair loss. The selective inhibition of JAK3 over other kinases may lead to a more favorable adverse effect profile than other JAK1/JAK2-panJAK inhibitors. Additional studies for long-term effects are warranted.

\section{Author Contributions}

All authors made substantial contributions to conception and design, acquisition of data, or analysis and interpretation of data; took part in drafting the article or revising it critically for important intellectual content; agreed to submit to the current journal; gave final approval of the version to be published; and agree to be accountable for all aspects of the work. 


\section{Funding}

The authors received no specific funding for this work.

\section{Disclosure}

Dr. Antonella Tosti is a Consultant of DS Laboratories, Monat Global, Pfizer Thirty Madison, Lilly, Leo Pharmaceuticals, Bristol Myers Squibb, and P\&G; and President AHRS Editor SAD. Dr Ramírez-Marín has nothing to disclose.

\section{References}

1. Villasante Fricke AC, Miteva M. Epidemiology and burden of alopecia areata: a systematic review. Clin Cosmet Investig Dermatol. 2015;8:397-403. doi:10.2147/CCID.S53985.

2. Pratt CH, King LE Jr, Messenger AG, Christiano AM, Sundberg JP. Alopecia areata. Nat Rev Dis Primers. 2017;3:17011. doi:10.1038/ nrdp.2017.11.

3. Safavi K. Prevalence of alopecia areata in the First National Health and Nutrition Examination Survey. Arch Dermatol. 1992;128(5):702. doi:10.1001/archderm.1992.01680150136027.

4. National Alopecia Areata Foundation. What you need to know about alopecia areata; 2021. Available from: https://www.naaf.org/alopecia-areata. Accessed November 5, 2021.

5. Goh C, Finkel M, Christos PJ, Sinha AA. Profile of 513 patients with alopecia areata: associations of disease subtypes with atopy, autoimmune disease and positive family history. J Eur Acad Dermatol Venereol. 2006;20(9):1055-1060. doi:10.1111/j.1468-3083.

6. Gip L, Lodin A, Molin L. Alopecia areata. A follow-up investigation of outpatient material. Acta Derm Venereol. 1969;49(2):180-188.

7. Tosti A, Bellavista S, Iorizzo M. Alopecia areata: a long term follow-up study of 191 patients. J Am Acad Dermatol. 2006;55(3):438-441. doi:10.1016/j.jaad.2006.05.008.

8. De Waard-van der Spek FB, Oranje AP, De Raeymaecker DM, Peereboom-Wynia JD. Juvenile versus maturity-onset alopecia areata-a comparative retrospective clinical study. Clin Exp Dermatol. 1989;14(6):429-433. doi:10.1111/j.1365-2230.1989.tb02604.x.

9. Safavi KH, Muller SA, Suman VJ, Moshell AN, Melton LJ. Incidence of alopecia areata in Olmsted County, Minnesota, 1975 through 1989. Mayo Clin Proc. 1995;70(7):628-633. doi:10.4065/70.7.628

10. Hordinsky M, Junqueira AL. Alopecia areata update. Semin Cutan Med Surg. 2015;34(2):72-75. doi:10.12788/j.sder.2015.0160.

11. King B, Guttman-Yassky E, Peeva E, et al. A phase 2a randomized, placebo-controlled study to evaluate the efficacy and safety of the oral Janus kinase inhibitors ritlecitinib and brepocitinib in alopecia areata: 24-week results. $J$ Am Acad Dermatol. 2021;85(2):379-387. doi:10.1016/j. jaad.2021.03.050

12. O’Shea JJ, Murray PJ. Cytokine signaling modules in inflammatory responses. Immunity. 2008;28(4):477-487. doi:10.1016/j.immuni.2008.03.002.

13. Schwartz DM, Bonelli M, Gadina M, O'Shea JJ. Type I/II cytokines, JAKs, and new strategies for treating autoimmune diseases. Nat Rev Rheumatol. 2016;12(1):25-36. doi:10.1038/nrrheum.2015.167.

14. Rochman Y, Spolski R, Leonard WJ. New insights into the regulation of T cells by gamma(c) family cytokines. Nat Rev Immunol. 2009;9 (7):480-490. doi:10.1038/nri2580.

15. Xing L, Dai Z, Jabbari A, et al. Alopecia areata is driven by cytotoxic T lymphocytes and is reversed by JAK inhibition. Nat Med. 2014;20 (9):1043-1049. doi:10.1038/nm.3645.

16. El Aziz Ragab MA, Hassan EM, El Niely DAEM, Mohamed MM. Serum level of interleukin-15 in active alopecia areata patients and its relation to age, sex, and disease severity. Postepy Dermatol Alergol. 2020;37(6):904-908. doi:10.5114/ada.2020.102103.

17. Gilhar A, Etzioni A, Paus R. Alopecia areata. N Engl J Med. 2012;366(16):1515-1525. doi:10.1056/NEJMra1103442.

18. Gilhar A. Collapse of immune privilege in alopecia areata: coincidental or substantial? J Invest Dermatol. 2010;130(11):2535-2537. doi:10.1038/ jid.2010.260

19. Tzeng HT, Chyuan IT, Lai JH. Targeting the JAK-STAT pathway in autoimmune diseases and cancers: a focus on molecular mechanisms and therapeutic potential. Biochem Pharmacol. 2021;193:114760. doi:10.1016/j.bcp.2021.114760.

20. United States Food and Drug Administration. Coronavirus (COVID-19) Update: FDA authorizes drug combination for treatment of COVID-19. FDA news release; 2020. Available from: https://www.fda.gov/news-events/press-announcements/coronavirus-covid-19-update-fda-authorizes-drug -combination-treatment-covid-19. Accessed November 3, 2021.

21. Jabbari A, Sansaricq F, Cerise J, et al. An open-label pilot study to evaluate the efficacy of tofacitinib in moderate to severe patch-type alopecia areata, totalis, and universalis. J Invest Dermatol. 2018;138(7):1539-1545. doi:10.1016/j.jid.2018.01.032.

22. Kennedy Crispin M, Ko JM, Craiglow BG, et al. Safety and efficacy of the JAK inhibitor tofacitinib citrate in patients with alopecia areata. JCI Insight. 2016;1(15):e89776. doi:10.1172/jci.insight.89776.

23. Mackay-Wiggan J, Jabbari A, Nguyen N, et al. Oral ruxolitinib induces hair regrowth in patients with moderate-to-severe alopecia areata. JCI Insight. 2016;1(15):e89790. doi:10.1172/jci.insight.89790.

24. Liu LY, Craiglow BG, Dai F, King BA. Tofacitinib for the treatment of severe alopecia areata and variants: a study of 90 patients. $J$ Am Acad Dermatol. 2017;76(1):22-28. doi:10.1016/j.jaad.2016.09.007.

25. Craiglow BG, Liu LY, King BA. Tofacitinib for the treatment of alopecia areata and variants in adolescents. J Am Acad Dermatol. 2017;76 (1):29-32. doi:10.1016/j.jaad.2016.09.006.

26. Casella J. AAD 2019; S034, Abstract 11291.

27. Liu LY, King BA. Ruxolitinib for the treatment of severe alopecia areata. J Am Acad Dermatol. 2019;80(2):566-568. doi:10.1016/j. jaad.2018.08.040.

28. Clark JD, Flanagan ME, Telliez JB. Discovery and development of Janus kinase (JAK) inhibitors for inflammatory diseases. J Med Chem. 2014;57 (12):5023-5038. doi:10.1021/jm401490p.

29. Schwartz DM, Kanno Y, Villarino A, Ward M, Gadina M, O'Shea JJ. JAK inhibition as a therapeutic strategy for immune and inflammatory diseases. Nat Rev Drug Discov. 2017;16(12):84. 
30. Choy EH. Clinical significance of Janus Kinase inhibitor selectivity. Rheumatology. 2019;58(6):953-962. doi:10.1093/rheumatology/key339.

31. Dai Z, Chen J, Chang Y, Christiano AM. Selective inhibition of JAK3 signaling is sufficient to reverse alopecia areata. JCI Insight. 2021;6(7): e142205. doi:10.1172/jci.insight. 142205

32. Macchi P, Villa A, Giliani S, et al. Mutations of Jak-3 gene in patients with autosomal severe combined immune deficiency (SCID). Nature. 1995;377(6544):65-68. doi:10.1038/377065a0

33. Jo CE, Gooderham M, Beecker J. TYK 2 inhibitors for the treatment of dermatologic conditions: the evolution of JAK inhibitors. Int J Dermatol. 2021. doi:10.1111/ijd.15605

34. Xu H, Jesson MI, Seneviratne UI, et al. PF-06651600, a Dual JAK3/TEC family kinase inhibitor. ACS Chem Biol. 2019;14(6):1235-1242. doi:10.1021/acschembio.9b00188

35. Virtanen A, Haikarainen T, Raivola J, Silvennoinen O. Selective JAKinibs: prospects in inflammatory and autoimmune diseases. BioDrugs. 2019;33(1):15-32. doi:10.1007/s40259-019-00333-w

36. Berg LJ, Finkelstein LD, Lucas JA, Schwartzberg PL. Tec family kinases in T lymphocyte development and function. Annu Rev Immunol. 2005;23:549-600. doi:10.1146/annurev.immunol.22.012703.104743

37. Schwartzberg PL, Finkelstein LD, Readinger JA. TEC-family kinases: regulators of T-helper-cell differentiation. Nat Rev Immunol. 2005;5 (4):284-295. doi:10.1038/nri1591

38. Andreotti AH, Schwartzberg PL, Joseph RE, Berg LJ. T-cell signaling regulated by the Tec family kinase, Itk. Cold Spring Harb Perspect Biol. 2010;2(7):a002287. doi:10.1101/cshperspect.a002287

39. Kurosaki T. Regulation of BCR signaling. Mol Immunol. 2011;48(11):1287-1291. doi:10.1016/j.molimm.2010.12.007

40. Selleckchek.com. Ritlecitinib (PF-06651600); 2021. Available from: https://www.selleckchem.com/products/pf-06651600.html. Accessed November 3, 2021.

41. NCIthesaurus. Ritlecitinib; 2021. Available from: https://ncithesaurus.nci.nih.gov/ncitbrowser/pages/concept_details.jsf?dictionary=NCI_ Thesaurus\&version $=21.10 \mathrm{~d} \&$ code $=\mathrm{C} 161783 \not$ ncit\&type $=$ properties $\&$ key $=$ null\&b=1\&n=0\&vse $=$ null. Accessed November 3, 2021.

42. Telliez JB, Dowty ME, Wang L, et al. Discovery of a JAK3-selective inhibitor: functional differentiation of JAK3-selective inhibition over pan-JAK or JAK1-selective inhibition. ACS Chem Biol. 2016;11(12):3442-3451. doi:10.1021/acschembio.6b00677

43. Robinson MF, Damjanov N, Stamenkovic B, et al. Efficacy and Safety of PF-06651600 (Ritlecitinib), a Novel JAK3/TEC inhibitor, in patients with moderate-to-severe rheumatoid arthritis and an inadequate response to methotrexate. Arthritis Rheumatol. 2020;72:1621-1631.

44. ClinicalTrials.gov. Study to evaluate the efficacy and safety profile of PF-06651600 and PF-06700841 in subjects with alopecia areata; 2020. Availabe from: https://clinicaltrials.gov/ct2/show/NCT02974868. Accessed November 5, 2021.

45. Peeva E, Banerjee A. Oral Janus kinase inhibitors PF-06700841 and PF-06651600 provide clinically evident therapeutic effect at 4 and 6 weeks in patients with alopecia areata and greater efficacy over 24 weeks in patients with a shorter duration of their current alopecia episo. American Academy of Dermatology Annual Meeting; 2019; Washington, DC, USA.

46. ClinicalTrials.gov. Placebo-controlled safety study of ritlecitinib (PF-06651600) in adults with alopecia areata (Allegro2a); 2021. Available from: https://clinicaltrials.gov/ct2/show/NCT04517864. Accessed November 2, 2021.

47. ClinicalTrials.gov. PF-06651600 for the treatment of alopecia areata (ALLEGRO-2b/3); 2021. Available from: https://clinicaltrials.gov/ct2/show/ NCT03732807? id $=$ NCT03732807\&draw=2\&rank=1. Accessed November 2, 2021.

48. Pfizer. Pfizer announces positive top-line results from Phase $2 b / 3$ trial of ritlecitinib in alopecia areata. Press release; 2021. Available from: https://www.pfizer.com/news/press-release/press-release-detail/pfizer-announces-positive-top-line-results-phase-2b3-trial. Accessed November $11,2021$.

49. Olsen EA, Hordinsky MK, Price VH, et al. Alopecia areata investigational assessment guidelines-Part II. National Alopecia Areata Foundation. $J$ Am Acad Dermatol. 2004;51(3):440-447. doi:10.1016/j.jaad.2003.09.032

50. ClinicalTrials.gov. Long-term PF-06651600 for the treatment of alopecia areata (ALLEGRO-LT); 2021. https://clinicaltrials.gov/ct2/show/ NCT04006457. Accessed November 2, 2021.

51. Peeva E, Banerjee A, Guttman-Yassky E, et al. Safety and Efficacy of the Oral JAK3/TEC Inhibitor PF-06651600 and the Oral TYK2/JAK1 Inhibitor PF-06700841 in a withdrawal/re-treatment extension study in patients with alopecia areata. Presented at the 28th Congress of the European Academy of Dermatology and Venereology.

52. Evangelatos G, Koulouri V, Iliopoulos A, Fragoulis GE. Tuberculosis and targeted synthetic or biologic DMARDs, beyond tumor necrosis factor inhibitors. Ther Adv Musculoskelet Dis. 2020;12:1759720X20930116. doi:10.1177/1759720X209301

53. Sunzini F, McInnes I, Siebert S. JAK inhibitors and infections risk: focus on herpes zoster. Ther Adv Musculoskelet Dis. 2020;12:1759720X20936059. doi:10.1177/1759720X20936059

54. Schreiber RD, Old LJ, Smyth MJ. Cancer immunoediting: integrating immunity's roles in cancer suppression and promotion. Science. 2011;331 (6024):1565-1570. doi:10.1126/science.1203486

55. Harigai M. Growing evidence of the safety of JAK inhibitors in patients with rheumatoid arthritis. Rheumatology. 2019;58(Suppl 1):i34-i42. doi:10.1093/rheumatology/key287

56. Khan S. How do jak inhibitors (DMARDS) work?; 2021. Available from: https://www.rxlist.com/how_do_jak_inhibitors_dmards_work/drug-class. htm. Accessed November 3, 2021.

57. Fujita Y, Matsuoka N, Temmoku J, et al. JAK inhibitors impair GM-CSF-mediated signaling in innate immune cells. BMC Immunol. 2020;21 (1):35. doi:10.1186/s12865-020-00365-w

58. Fragoulis GE, McInnes IB, Siebert S. JAK-inhibitors. New players in the field of immune-mediated diseases, beyond rheumatoid arthritis. Rheumatology. 2019;58(Supp11):i43-i54. doi:10.1093/rheumatology/key276

59. Rajasimhan S, Pamuk O, Katz JD. Safety of janus kinase inhibitors in older patients: a focus on the thromboembolic risk. Drugs Aging. 2020;37 (8):551-558. PMID: 32514874; PMCID: PMC7387323. doi:10.1007/s40266-020-00775-w

60. Administration USF and D. FDA requires warnings about increased risk of serious heart-related events, cancer, blood clots, and death for JAK inhibitors that treat certain chronic inflammatory conditions; 2021.

61. Peeva E, Craiglow B, Banerjee A, et al. A Phase 2a randomized, placebo-controlled study to evaluate efficacy and safety of janusfi kinase inhibitors PF-06651600 and PF-06700841 in alopecia areata: 24-week results. Presented at the 27th Congress of the Europea. 


\section{Publish your work in this journal}

Drug Design, Development and Therapy is an international, peer-reviewed open-access journal that spans the spectrum of drug design and development through to clinical applications. Clinical outcomes, patient safety, and programs for the development and effective, safe, and sustained use of medicines are a feature of the journal, which has also been accepted for indexing on PubMed Central. The manuscript management system is completely online and includes a very quick and fair peer-review system, which is all easy to use. Visit http://www.dovepress.com/testimonials.php to read real quotes from published authors.

Submit your manuscript here: https://www.dovepress.com/drug-design-development-and-therapy-journal 